\title{
Linear and Helical Cesium lodide Atomic Chains in Ultranarrow Single-Walled Carbon Nanotubes: Impact on Optical Properties
}

\author{
Reza J. Kashtiban,* Maria G. Burdanova, Andrij Vasylenko,* Jamie Wynn, Paulo V. C. Medeiros,
} Quentin Ramasse, Andrew J. Morris, David Quigley, James Lloyd-Hughes, and Jeremy Sloan*

Cite This: ACS Nano 2021, 15, 13389-13398

Read Online

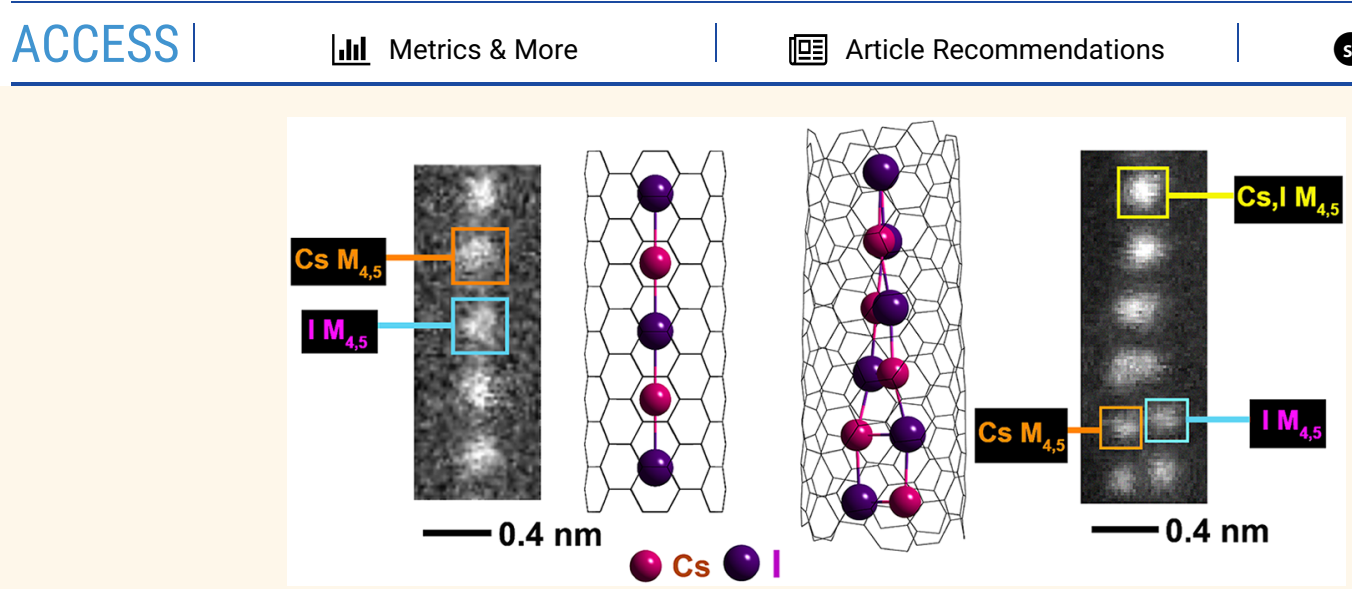

ABSTRACT: One-dimensional (1D) atomic chains of CsI were previously reported in double-walled carbon nanotubes with $\sim 0.8 \mathrm{~nm}$ inner diameter. Here, we demonstrate that, while 1D CsI chains form within narrow $\sim 0.73$ $\mathrm{nm}$ diameter single-walled carbon nanotubes (SWCNTs), wider SWCNT tubules $(\sim 0.8-1.1 \mathrm{~nm})$ promote the formation of helical chains of CsI $2 \times 1$ atoms in cross-section. These CsI helices create complementary oval distortions in encapsulating SWCNTs with highly strained helices formed from strained $\mathrm{Cs}_{2} \mathrm{I}_{2}$ parallelogram units in narrow tubes to lower strain $\mathrm{Cs}_{2} \mathrm{I}_{2}$ units in wider tubes. The observed structural changes and charge distribution were analyzed by density-functional theory and Bader analysis. CsI chains also produce conformation-selective changes to the electronic structure and optical properties of the encapsulating tubules. The observed defects are an interesting variation from defects commonly observed in alkali halides as these are normally associated with the Schottky and Frenkel type. The energetics of CsI $2 \times 1$ helix formation in SWCNTs suggests how these could be controllably formed.

KEYWORDS: atomic chains, carbon nanotubes, electron microscopy, density functional theory Raman spectroscopy,

photoluminescence

"True" one-dimensional (1D) atomic chains were first formed by relocating atoms with a scanning tunnelling microscope (STM) tip under cryogenic conditions, ${ }^{1-4}$ demonstrating quantum scale device fabrication, albeit with a one-off approach that limited studies to the laboratory. More recently, $\mathrm{M}_{1-x} \mathrm{M}^{\prime}{ }_{x} \mathrm{X}_{1-y} \mathrm{X}^{\prime}{ }_{y}\left(\mathrm{M}\right.$ or $\mathrm{M}^{\prime}=\mathrm{Mo}$ or $\mathrm{W}$ and $\mathrm{X}^{\prime}=\mathrm{S}$, Se, Te, or I) $1 \mathrm{D}$ nanowires and nanowire junctions were formed by electron beam lithography from the corresponding $\mathrm{M}_{1-x} \mathrm{M}^{\prime}{ }_{x}\left(\mathrm{X}_{1-y} \mathrm{X}_{y}^{\prime}\right)_{2} 2 \mathrm{D}$ dichalcogenides. ${ }^{5-8}$ Templating atomic chain formation in carbon nanotubes, however, offers by far the greatest compositional diversity and scalability for 1D materials fabrication observed so far. Nanotube insertion occurs from the liquid or vapor phase and its van Der Waals surface templates integral, ${ }^{9}$ helical $^{10-12}$ or "zigzag"13 atomic columns in cross section, and in the limit, "true" 1D atomic chains in the narrowest tubules, ${ }^{12-26}$ although the characterization of single element materials including Se, Te, and $S$ in porous materials in

Received: May 1, 2021

Accepted: July 22, 2021

Published: August 9, 2021

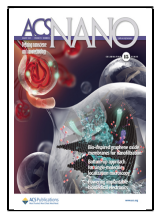


a

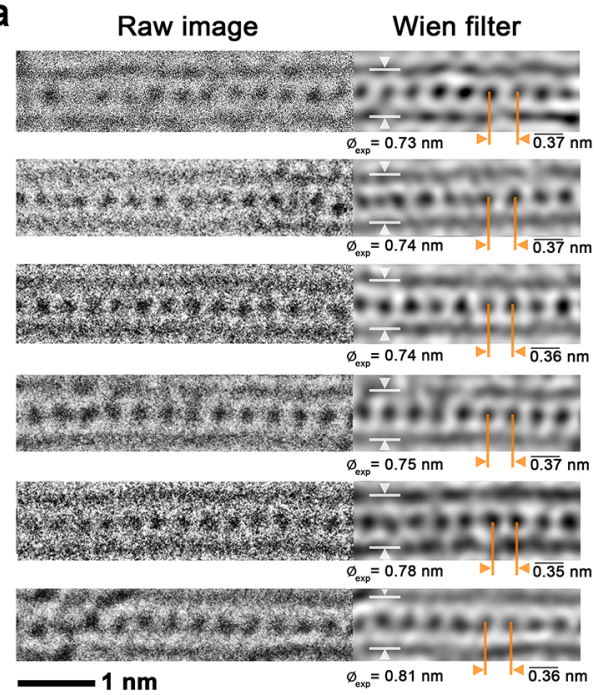

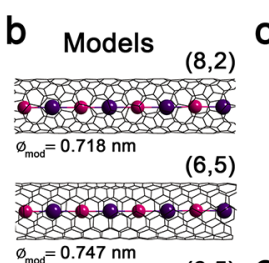

$(6,5)$
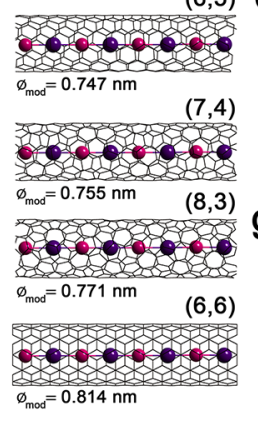

C

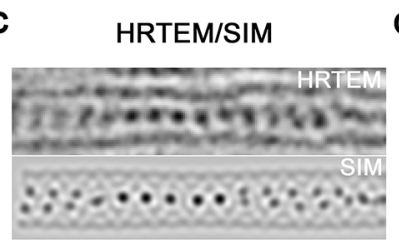

$\bar{\varnothing}=0.88 \mathrm{~nm}$ Cs- $\mathrm{I}_{\mathrm{av}}=0.37 \mathrm{~nm}$.

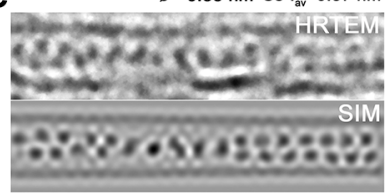

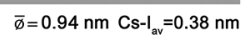

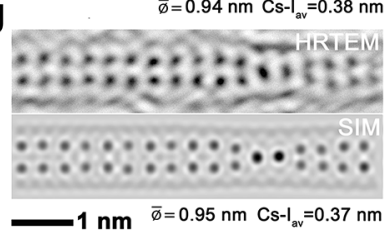

d

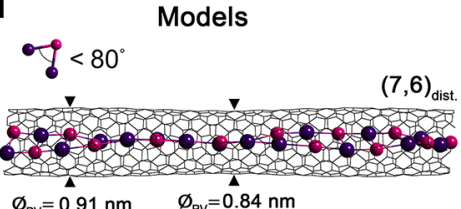

$\emptyset_{\mathrm{PV}}=0.91 \mathrm{~nm} \quad \emptyset_{\mathrm{PV}}=0.84 \mathrm{~nm}$

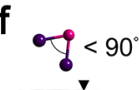

$(7,7)_{\text {dist. }}$
$\emptyset_{\mathrm{PV}}=0.99 \mathrm{~nm} \quad \emptyset_{\mathrm{PV}}=0.90 \mathrm{~nm}$

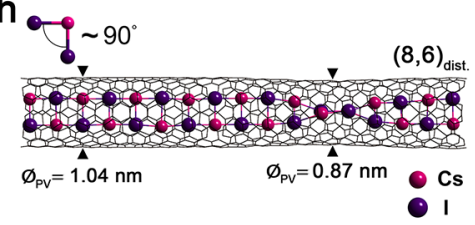

Figure 1. (a) Six HRTEM images and Wien-filtered details of linear single atomic width Cs-I chains in SWCNTs with measured diameters increasing from 0.73 to $0.81 \mathrm{~nm}$. (b) Structure models of Cs-I linear chains inside SWCNTs with $(n, m)$ conformations specifying diameters matched to the composites imaged in a. $(c-h)$ HRTEM images, corresponding composite structure models and multislice image simulations (denoted SIM) of twisted Cs-I $2 \times 1$ atomic chains in SWCNTs spanning $(c, d)$ average $0.88 \mathrm{~nm}$ diameter, $(\mathrm{e}, \mathrm{f})$ average $0.94 \mathrm{~nm}$ diameter and $(\mathrm{g}, \mathrm{h})$ average $0.95 \mathrm{~nm}$ diameter. All three nanotubes distort $\sim 10 \%$ in the cross section in response to the twisting of the embedded $2 \times 1$ atomic chains (see also Figure $2 \mathrm{a}-\mathrm{f}$ ) but, as the diameter of the encapsulating SWCNT widens the internal I-Cs-I bond angles relax from $\sim 72-83^{\circ}$ (i.e., c,d and e,f) to $\sim 90^{\circ}$ as indicated by the inset motifs.

aligned porous materials such as mordenite and $\mathrm{AlPO}_{4}$ have also been extensively reported. .,27,28 $^{-1}$

Several groups have demonstrated 1D and helical iodine chain formation in single-walled carbon nanotubes (SWCNTs) ${ }^{10,15}$ and double-walled carbon nanotubes (DWCNTs) ${ }^{16,17}$ and linear chains of $S,{ }^{12}$ carbyne, ${ }^{17} \mathrm{Te}^{18} \mathrm{P}^{19}$ and $\mathrm{As}^{20}$ in narrow SWCNTs. Functionality can be extended if the nanowires are formed from binary or ternary phases, and Senga et al. demonstrated linear chains (or "true" $1 \mathrm{D}$ crystals) of $\mathrm{CsI}^{13}$ and NaI, LiI, CsF, and $\mathrm{Cs}_{1-x} \mathrm{~K}_{x}$ I within DWCNTs. ${ }^{23}$ Binary $1 \mathrm{D}$ chain filling in narrow SWCNTs was also demonstrated for chalcogenides $\mathrm{SnTe}^{21}$ and $\mathrm{SnSe}^{22}$ for which computations with density-dunctional theory (DFT) indicated enhanced thermoelectric properties in isolated nanowires of the former. 1D properties have been investigated by Raman spectroscopy for $\mathrm{I}_{3}{ }^{-}, \mathrm{I}_{5}{ }^{-24-26} \mathrm{KI}^{29}$ and HgTe. ${ }^{30}$ True $1 \mathrm{D}$ physical phenomena such as Peierls distortions (e.g., $\left.\mathrm{Te}_{x}\right)^{18}$ and Charge Density Waves (e.g., $\mathrm{I}_{x}^{-}$) can potentially also be addressed. ${ }^{15}$ More extrinsic properties of $1 \mathrm{D}$ crystal encapsulated SWCNT (i.e., 1D@SWCNT) composites include the fine-tuning of nanotube properties by doping or charge transfer. ${ }^{24-26,30-33}$ Calculations from first-principles suggest that $1 \mathrm{D}$ CsI crystals perturb the band structure of the narrowest SWCNTs with fluctuations in the electron density in the CNT commensurate with the periodicity of the CsI chain and which diminish as the encapsulating diameter increases and/or the 1D structure deviates from linearity. ${ }^{14}$

Our investigation establishes the precise experimental structural evolution of atomic chains of CsI formed inside narrow SWCNTs with diameters of $0.7-1.1 \mathrm{~nm}$, producing structures that vary from "true" $1 \mathrm{D} \mathrm{CsI,} \mathrm{to} \mathrm{highly} \mathrm{strained} 2 \times 1$ CsI helices with diameter. We imaged these structures using a combination of high-resolution transmission electron microscopy (HRTEM) and annular dark field (ADF) in scanning transmission electron microscopy (STEM) imaging mode and also atomically resolved electron energy loss spectroscopic
(EELS) imaging. We reveal by Raman, optical absorption, and photoluminescence spectroscopy that the structural distortions of the SWCNTs induced by the CsI filling selectively modify the optoelectronic properties of tubules with specific $(n, m)$ chiralities.

\section{RESULTS AND DISCUSSION}

Samples of CsI-filled SWCNTs were produced by filling $20 \mathrm{mg}$ quantities of preoxidized SWeNT nanotubes by sublimation using an adapted procedure ${ }^{13}$ as described in the Methods. Preliminary imaging studies were performed at $80 \mathrm{kV}$ in a double aberration corrected JEOL ARM200F instrument operated in phase contrast HRTEM and annular dark field (ADF) imaging modes. Further high angle annular dark field (HAADF) imaging studies were performed at $60 \mathrm{kV}$ in a Nion UltraSTEM with EELS being recorded on a Gatan Enfina spectrometer, which also enabled the acquisition of atomically resolved Cs and I chemical maps with a $20 \mathrm{pA}$ electron beam current and $\sim 0.1 \mathrm{~nm}$ resolution. Further experimental details are recorded in the Methods.

HRTEM, ADF Imaging, and EELS Mapping. Figure 1 shows the evolution of the CsI microstructure with SWCNT cross-section for a SWCNTs ranging in diameter from 0.73 to $0.95 \mathrm{~nm}$ as imaged in plan view by aberration-corrected HRTEM. In the diameter range $0.73-0.81 \mathrm{~nm}$ (Figure 1a,b), linear -Cs-I-Cs-I- atomic chains are observed with an average interatomic separation of $\sim 0.36 \mathrm{~nm}$, comparable to the $\sim 0.34 \mathrm{~nm}$ separation recorded within CsI-filled DWCNTs ${ }^{13}$ with innermost SWCNT diameters as small as $\sim 0.80 \mathrm{~nm}$. When the imaged SWCNT has a diameter just larger than $\sim 0.87 \mathrm{~nm}$ (Figure 1c,d), a distortion of both the 1D crystal and the nanotube is observed in which the crystal forms a twisting $2 \times 1$ "zigzag" configuration with $-\mathrm{I}-\mathrm{Cs}-\mathrm{I}-$ internal angle of $\sim 71^{\circ}$ with a loss of linear symmetry. A $\sim 10 \%$ oval distortion in the SWCNT cross section is observed coincident with the $2 \times 1$ nanostructure twist, comparable to distortions reported for 
a
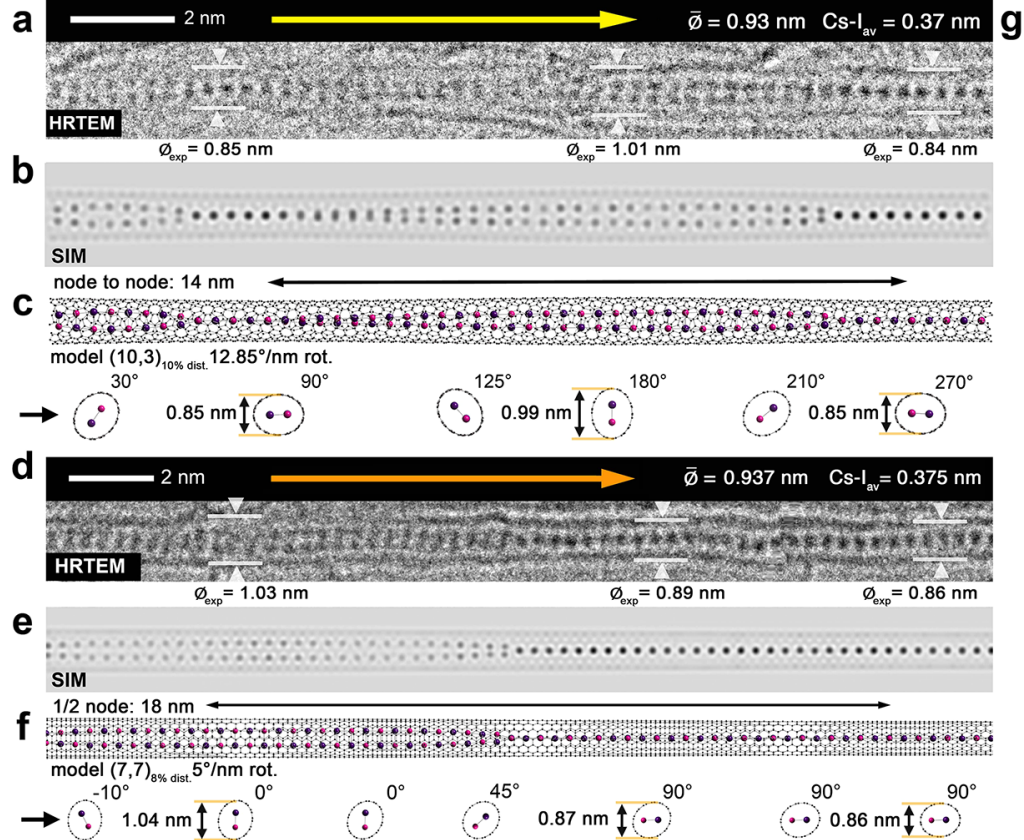

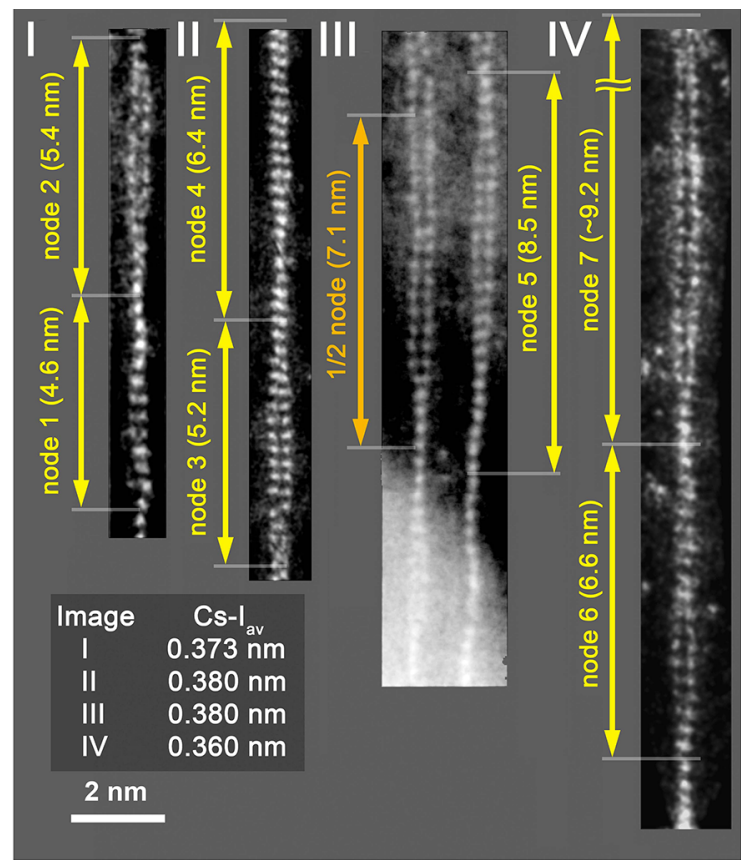

Figure 2. $(\mathrm{a}-\mathrm{c})$ HRTEM lattice image, multislice simulation and a model of a $2 \times 1$ helical chain of CsI formed inside a distorted $\sim 0.90 \mathrm{~nm}$ average diameter SWCNT. The $\sim 14 \mathrm{~nm}$ pitch and distortion can be seen by viewing $(\mathrm{a}-\mathrm{c})$ at a glancing angle in the direction indicated. The relative orientation of the two-atom CsI unit in ovally distorted SWCNTs relative to the electron beam (black arrow) is given by cross-sectional models at the bottom of $c$. $(d-f)$ HRTEM lattice image, multislice simulation, and corresponding models of a longer $\sim 36 \mathrm{~nm}$ pitch $2 \times 1$ helical chain imaged inside a $\sim 0.94 \mathrm{~nm}$ diameter SWCNT, assignations as for $(\mathrm{a}-\mathrm{c})$. (g) Four ADF STEM images $(\mathrm{I}-\mathrm{IV})$ of five CsI $2 \times 1$ helical chains inside SWCNTs showing the variability in pitch, even within a single SWCNT. Cs $-I$ atomic distances $\left(C s-I_{\text {av }}\right)$, measured longitudinally for all micrographs (i.e., (a), (d), ( $(\mathrm{I}-\mathrm{IV})))$, average in the range $0.36-0.38 \mathrm{~nm}$. Note that for all images, whenever the electron beam passes through a two-atom column, we see a doubling of the observed atom contrast.

SWCNT encapsulating quasi-1D chains of iodine, ${ }^{10,15} \mathrm{HgI}_{2}{ }^{11}$ and $\mathrm{CoI}_{2} \cdot{ }^{34}$ The SWCNT in Figure $1 \mathrm{c}$, d has an average diameter of $0.88 \mathrm{~nm}$ with narrower $\sim 0.81$ and wider $\sim 0.91 \mathrm{~nm}$ sections visible. In a second example (Figure 1e,f), a SWCNT is filled with $2 \times 1 \mathrm{CsI}$ in which the average diameter increases to 0.94 $\mathrm{nm}$ with an increase in the I-Cs-I angle to $\sim 75^{\circ}$. In a third example (Figure 1g,h), a $2 \times 1$ CsI chain with undistorted $90^{\circ}$ internal I-Cs-I angle is observed, but it exhibits a short $180^{\circ}$ helical twist defect with a corresponding distortion in the SWCNT (average diameter $\sim 0.95 \mathrm{~nm}$ ). In the above, local variations in the local imaged plan view diameter are sometimes seen as a result of debris on the SWCNTs, knock-on damage, or radiolysis, but the overall fluctuations in diameter are smooth.

In Figure $2 \mathrm{a}-\mathrm{g}$, seven $2 \times 1 \mathrm{CsI}$ chains imaged by HRTEM (Wien-filtered) and ADF STEM with evident helical symmetry are presented. Viewing each imaged object at glancing angles in the indicating arrowed directions enables the helicity to be more easily visualized. The multislice simulations in Figure $2 b$,e are based on the corresponding models in Figure 2c,f, respectively, with the orientation of a two-atom CsI unit with respect to the electron beam at each horizontal position indicated below. The pitch of the helices imaged in Figure 2 varies from $\sim 5 \mathrm{~nm}$ (Figure $2 \mathrm{gII}$ ) to $\sim 36 \mathrm{~nm}$ (Figure $2 \mathrm{~d}$ ). The Cs-I distances, averaged longitudinally along each helical chain, vary from 0.36 $\mathrm{nm}$ up to $0.38 \mathrm{~nm}$, comparable to those recorded by Senga et al. ${ }^{13}$ but significantly longer than the I-I distances reported for charged iodine chains (see also Figure $5 \mathrm{~d}-\mathrm{f}$ ). ${ }^{10,14-16}$

Confirmation of the chemistry and local stoichiometry of both linear and helical chain CsI filling is given in Figure $3 a-i$. Aberration-corrected HAADF images and atomically resolved elemental EELS maps and matching models are presented for a linear $-\mathrm{I}-\mathrm{Cs}-\mathrm{I}-\mathrm{Cs}-\mathrm{I}-\mathrm{chain}$ (Figure $3 \mathrm{a}-\mathrm{c}$ ) and for a helical 2 $\times 1 \mathrm{Cs}-\mathrm{I}$ chain (Figure $3 \mathrm{e}-\mathrm{g}$ ). EEL spectra (Figure 3d) obtained from the boxed regions in Figure $3 \mathrm{~b}$ show $\mathrm{M}_{4,5}$ edges from discrete $\mathrm{Cs}$ and $\mathrm{I}$ atoms corresponding to the $-\mathrm{I}-\mathrm{Cs}-\mathrm{I}-$ Cs-I- model (Figure 3c). An EEL spectrum, obtained from an imaged Cs-I atom pair with doubled $\mathrm{Z}$ contrast (Figure $3 \mathrm{f}$ ), shows both $\mathrm{Cs}$ and $\mathrm{I} \mathrm{M}_{4,5}$ edges (Figure $3 \mathrm{~h}$ ), whereas discrete atoms indicated by the boxes in the lower part of Figure $3 \mathrm{f}$ produce separate $\mathrm{Cs}$ and $\mathrm{I}_{4,5}$ edges (Figure $3 \mathrm{i}$ ) in good agreement with the perspective helical model in Figure $3 \mathrm{~g}$. Average diameters of the SWCNTs in Figure 3a,e are 0.78 and $0.91 \mathrm{~nm}$, respectively. Average Cs $-\mathrm{I}$ (i.e., $\mathrm{Cs}-\mathrm{I}_{\mathrm{av}}$ ) spacings for linear or helical $2 \times 1$ chains along SWCNTs were measured by direct imaging, by either fast Fourier transforms (FFTs) ${ }^{13}$ calculated from HRTEM images or direct electron diffraction in both cases obtained from aligned filled SWCNT bundles. ${ }^{35}$ Direct imaging measurements averaged over $2010-20 \mathrm{~nm}$ long CsI linear chains reveal a $\mathrm{Cs}-\mathrm{I}_{\mathrm{av}}$ of $\sim 0.36 \mathrm{~nm}$. Cs $-\mathrm{I}_{\mathrm{av}}$ lengthens to $\sim 0.37 \mathrm{~nm}$ for a similar number of measured $2 \times 1$ helical Cs-I chains, reflecting the somewhat higher strain in the latter. FFTs obtained from bundles of SWCNTs filled with CsI (Figure $\mathrm{S} 2 \mathrm{a}, \mathrm{b})$ reveal some variability in $\mathrm{Cs}-\mathrm{I}_{\mathrm{av}}$ from bundle to bundle, which indicates population differences in linear 1D CsI chains versus $2 \times 1 \mathrm{Cs}-\mathrm{I}$ chains in these composites.

DFT Modeling and Bader Analysis. The structures of atomic chains of CsI in varying degrees of confinement can be explored by DFT ${ }^{36,37}$ (Supporting Information) combined with charge distribution analyzed by Bader analysis. ${ }^{38}$ We modeled the isolated CsI $1 D$ wires initially from relaxed and stretched ion approaches, applying chain perturbations and examining the efficiency of the calculations using finite chains. A basic force- 


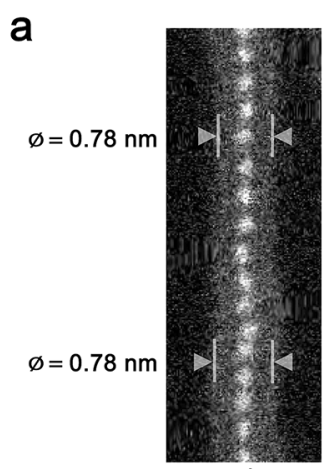

e $\quad-1 \mathrm{~nm}$

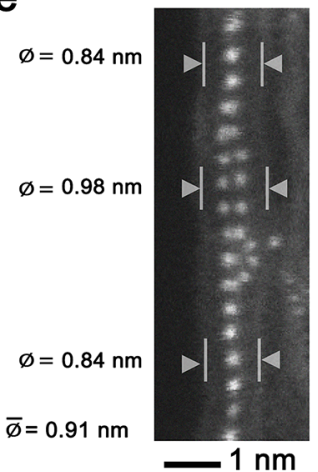

b

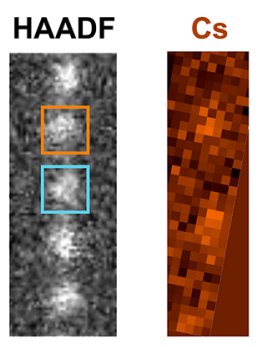

$-0.5 \mathrm{~nm}$

$f$

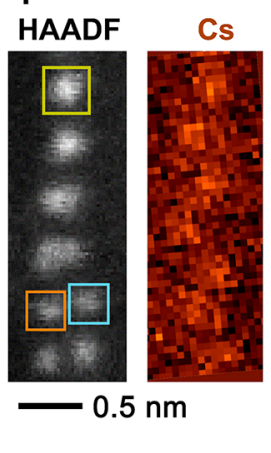

c
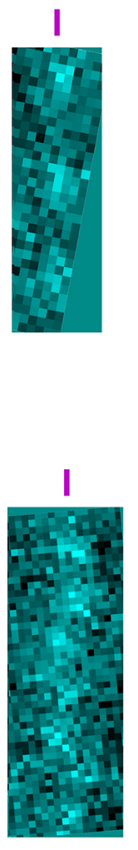

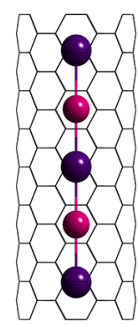

$(6,6)$
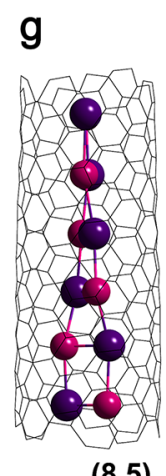

$(8,5)$ d

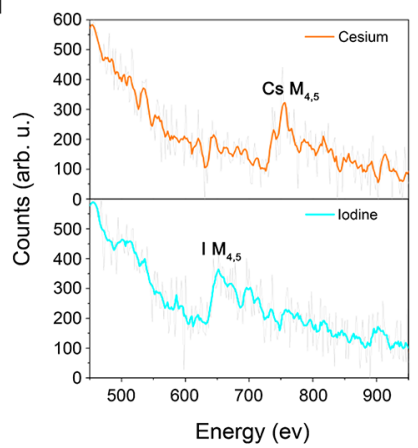

h

i

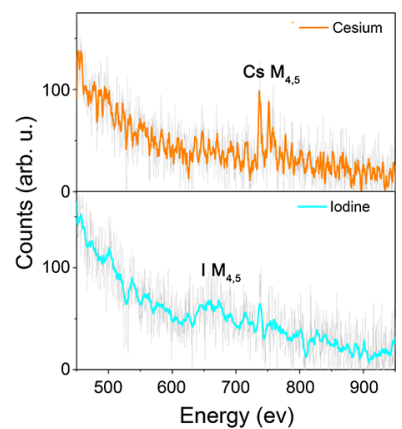

Figure 3. (a) HAADF image of a discrete CsI atomic chain imaged in a $\sim 0.78 \mathrm{~nm}$ diameter SWCNT. (b) HAADF detail and corresponding Cs and I EELS chemical maps showing alternating Cs and I. (c) Representative model of a 1D CsI atomic chain in an undistorted (6,6) SWCNT. (d) Cs and I $M_{4,5}$ EEL spectra obtained from the 2nd and 3rd atom, respectively, according to the indicating boxes in (b). (e) HAADF image of a rotating $2 \times 1$ helical chain imaged in a $\sim 10 \%$ sympathetically distorting $\sim 0.91 \mathrm{~nm}$ (average diameter) SWCNT. (f) HAADF detail and corresponding Cs and $\mathrm{I}_{4,5}$ EELS chemical maps showing coincident $\mathrm{Cs}$ and $\mathrm{I}$ atom pairs (top) and discrete CsI 1D chains (bottom) and Cs and I EELS chemical maps. (g) Perspective structure model of rotating $2 \times 1$ helical chain corresponding to the HAADF detail in (f). (h) EEL spectra obtained from a single $2 \times 1$ atom column obtained from the yellow boxed region in (f) in which both the Cs and I $M_{4,5}$ edges can clearly be seen. (i) CsI $M_{4,5}$ spectra obtained from the two discrete Cs and I atoms and indicated by the orange and blue boxed regions in (f).
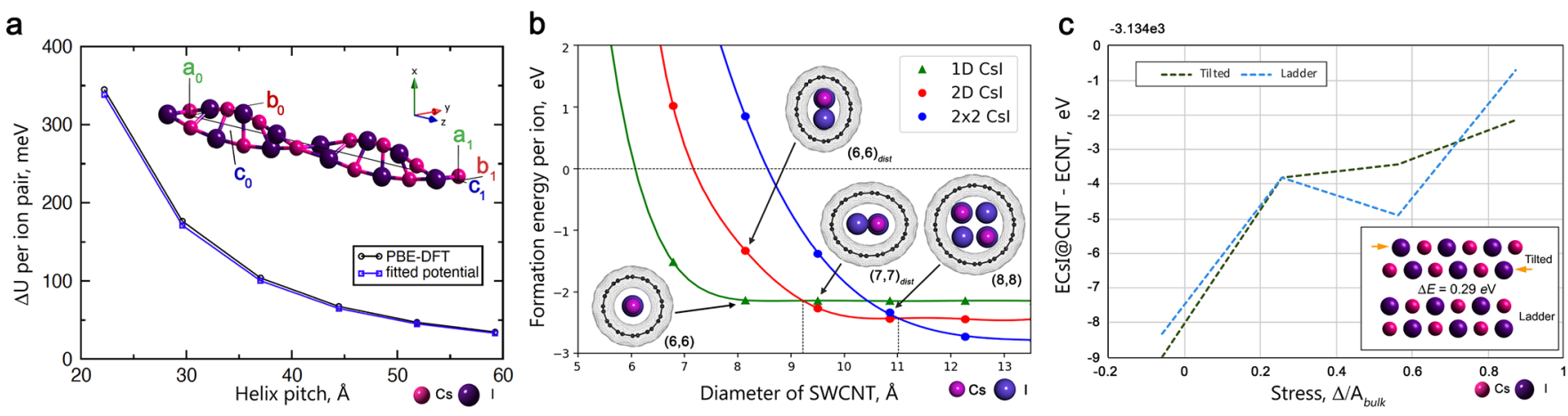

Figure 4. (a) Energy change in a twisting $2 \times 1$ chain of alternating dipoles into a helix of varying pitch. Results for both PBE-DFT and the fitted potential (parameter set 2, see the SI). Inset: dipole helix with 12 ion pairs. (b) Formation energy $\left(E_{\mathrm{f}}\right)$ of encapsulated CsI in SWCNTs calculated per atom energy difference between a formed complex structure $\left(E_{c}\right)$, a pristine SWCNT $\left(E_{\mathrm{CNT}}\right)$, and the sum of the chemical potentials of $\mathrm{Cs}$ and $\mathrm{I}$ atoms in a mixture of noninteracting atomic gases, $E_{\mathrm{at}}\left(\right.$ i.e., $\left.E_{\mathrm{f}}=E_{\mathrm{c}}-E_{\mathrm{CNT}}-E_{\mathrm{at}}\right)$ and plotted versus effective SWCNT diameter and averaged for an ovally distorted SWCNT. (c) Plot showing the difference in energy of a "Tilted" $2 \times 1$ CsI chain with $-I-C s-I-$ bond angles $<90^{\circ}$ versus a "Ladder" $2 \times 1 \mathrm{CsI}$ chain with $-\mathrm{I}-\mathrm{Cs}-\mathrm{I}-$ bond angles $\sim 90^{\circ}$ versus stress relative to the bulk.

field approach was utilized to examine different twisting conformations, summarized in Figure 4a, with which the fitted potential of a twisted helical $2 \times 1 \mathrm{Cs}-\mathrm{I}$ chain is compared with the same result established by DFT incorporating the PerdewBurke-Ernzerhof (PBE) functional. ${ }^{37}$ At the DFT level of accuracy, we then established the dependence of the formation energy of atomic chains of CsI as a function of the average diameter of undistorted and ovally distorted SWCNTs, respectively (Figure 4b, see also Figure S13b). A comparison of the energy trends for the experimentally observed linear -I-
Cs-I-Cs-I- and $2 \times 1 \mathrm{CsI}$ chains reveals a minimum $\sim 0.82$ $\mathrm{nm}$ SWCNT average diameter (note: in the case of a $2 \times 1 \mathrm{CsI}$ chain, the numerical "average" diameter of the encapsulating oval SWCNT corresponds to the numerical mean of its narrowest and widest measured diameter (i.e., Figure $1 \mathrm{c}-\mathrm{h}$ and Figure $2 a-f$ ) in which the formation of both is energetically possible but the linear $-\mathrm{I}-\mathrm{Cs}-\mathrm{I}-\mathrm{Cs}-\mathrm{I}-$ is energetically preferred (Figure 4b). We next identify a SWCNT diameter of $\sim 0.92 \mathrm{~nm}$ in which linear CsI chains coexist with highly strained zigzag $2 \times 1 \mathrm{CsI}$ chains with reduced I-Cs-I angles 
(green and red curves, Figure 4b). Above $\sim 0.92 \mathrm{~nm}$ diameter, relaxed $2 \times 1 \mathrm{CsI}$ chains with $\sim 90^{\circ}$ internal I-Cs-I angles (red curve, Figure $4 \mathrm{~b}$ ) in SWCNTs, in good agreement with experimental results (Figure $1 \mathrm{c}-\mathrm{h}$ ). In Figure $\mathrm{S} 13 \mathrm{~b}$, the formation energy study is extended to the next structure in the series, $2 \times 2$ CsI, for which the diameter crossover is $\sim 1.05 \mathrm{~nm}$.

The expected C, Cs, and I charge redistributions for linear (1D) Cs-I chains and $2 \times 1 \mathrm{CsI}$ as a function of encapsulation in narrow to wide armchair $(n, n)$ SWCNTs predicted by Bader analysis are presented in Table 1 . Linear $-\mathrm{I}-\mathrm{Cs}-\mathrm{I}-\mathrm{Cs}-\mathrm{I}-$

Table 1. Bader Charge Analysis in Terms of Outermost Valence Electrons and Formation energies $\left(E_{\mathrm{f}}\right)$ of Linear Chain 1D and $2 \times 1$ Chain 2D CsI@SWCNT

\begin{tabular}{|c|c|c|c|c|c|}
\hline & \multirow[b]{2}{*}{$\begin{array}{c}\text { SWCNT } \\
\text { diameter } \\
(\mathrm{nm})\end{array}$} & \multicolumn{3}{|c|}{$\begin{array}{l}\text { average population of } \\
\text { electronic orbitals }\end{array}$} & \multirow[b]{2}{*}{$\begin{array}{c}\text { formation } \\
\text { energy }\left(E_{\mathrm{f}}, \mathrm{eV}\right)\end{array}$} \\
\hline & & $\begin{array}{l}\mathrm{Cs},[] \\
5 \mathrm{~s} 5 \mathrm{p} 6 \mathrm{~s}\end{array}$ & $\begin{array}{l}\mathrm{I},[] \\
5 \mathrm{~s} 5 \mathrm{p}\end{array}$ & $C,[] 2 p$ & \\
\hline $\begin{array}{l}\text { 1D@ }(4,4) \\
\text { SWCNT }\end{array}$ & 0.525 & 7.196 & 8.270 & 4.011 & 3.261 \\
\hline $\begin{array}{l}\text { 1D@ } @(5,5) \\
\text { SWCNT }\end{array}$ & 0.678 & 7.662 & 8.200 & 3.994 & -1.517 \\
\hline $\begin{array}{r}\text { 1D@ }(6,6) \\
\text { SWCNT }\end{array}$ & 0.814 & 7.752 & 8.157 & 4.002 & -2.135 \\
\hline $\begin{array}{l}\text { 2D@(6,6) } \\
\text { SWCNT }\end{array}$ & 0.814 & 7.655 & 8.257 & 4.002 & -1.332 \\
\hline $\begin{array}{r}\text { 2D@(7,7) } \\
\text { SWCNT }\end{array}$ & 0.945 & 7.717 & 8.201 & 3.993 & -2.264 \\
\hline
\end{tabular}

chains encapsulated in very narrow SWCNTs (i.e., $(4,4)$ SWCNTs) are calculated to have a larger charge redistribution and higher formation energy than linear chains formed in $(5,5)$, $(6,6)$, and $(7,7)$ SWCNTs, suggesting mostly steric confinement of CsI within SWCNTs. In Figure 4c, using the electrostatic potential as steric confinement for an "implicit" $0.814 \mathrm{~nm}$ diameter $(6,6)$ SWCNT nanotube, ${ }^{18}$ we have studied that different degrees of stress may cause $2 \times 1$ CsI to vary in structure from a highly strained "tilted" $2 \times 1$ parallelogram form to a more relaxed or square-planar or "ladder" form as indicated by the inset structures. "Tilted" and "relaxed" $2 \times 1 \mathrm{CsI}$ ladder structures may coexist within $(6,6)$ SWCNTs with the latter being $\sim 3.8 \mathrm{meV} /$ atom lower in energy at $0 \mathrm{~K}$.

Dynamic Imaging, Comparison with lodine. Figure 5 reveals CsI dynamical imaging behavior in SWCNTs and images of the same tubules filled with iodine for comparative spectroscopic measurements. In Figures $5 \mathrm{a}, \mathrm{b}$ we see the motion of short 1D CsI fragments under the electron beam in straight and curved SWCNTs using $0.88-1.44 \mathrm{pA} / \mathrm{cm}^{2}$ e-beam dose. In both cases, short CsI chains move in a nonsmooth way along SWCNTs (Figure 5a,b; Movies S1 and S2). If a SWCNT is continuously filled with long CsI chains, little motion is observed (Figure 5c and Movie S3) and local deviations from the average Cs-I separation $\left(\mathrm{CsI}_{\mathrm{av}}\right)$ can be detected. In the straightened detail in Figure $5 c$, the Cs-I distance varies from $\sim 0.33$ to $\sim 0.37$ nm. For bundles of SWCNTs filled with iodine, we obtain similar average $\mathrm{I}-\mathrm{I}$ spacings (i.e., $\mathrm{I}-\mathrm{I}_{\mathrm{av}}$ ) to those reported within DWCNTs ${ }^{16}$ or narrow SWCNTs. ${ }^{10,15,16,25,26}$ Using the FFT protocol for imaged filled SWCNTs bundles (Figure $5 \mathrm{~d}$, inset), ${ }^{13,35}$ the $\mathrm{I}-\mathrm{I}_{\mathrm{av}}$ is $\sim 0.30 \mathrm{~nm}$. Iodine chains in individual SWCNTs show significant local differences, with some continuous $\mathrm{I}_{x}$ chains and $\sim 0.55-0.58 \mathrm{~nm}$ long $\mathrm{I}_{3}^{-}$trimers (Figure 5e). The extent to which trimer formation occurs in DWCNTs was reported to increase as the inner SWCNT diameter increased from 0.73 to $0.89 \mathrm{~nm},{ }^{15}$ consistent with our results. A schematic comparison of the relative microstructure of $\mathrm{I}_{x}$ chains versus CsI linear chains is shown in parts $\mathrm{f}$ and $\mathrm{g}$, respectively, of Figure 5.

Raman, Optical Absorption, and Photoluminescence Measurements. Pristine, CsI-filled, and I-filled SWCNT samples were characterized by Raman, optical-absorption spectroscopy (OAS), and photoluminescence (PL) spectroscopies, allowing $(n, m)$ chiral distributions and changes in electronic structure to be evaluated for filled $(6,4)$ to $(10,6)$ SWCNTs, with outlier contributions from wider SWNTs. Resonant Raman spectroscopy was performed at 532 and 660 $\mathrm{nm}$ (Figures 6a, S14c, and S14a,b) for pristine, CsI-filled, and iodine-filled SWCNTs. ${ }^{5}$ The 266 and $275 \mathrm{~cm}^{-1}$ radial breathing mode (RBM) peaks in unfilled, CsI-filled, and iodine-filled SWCNTs can be assigned to $(7,6)$ and $(8,4)$ tubes, respectively. ${ }^{39-41}$ More striking are three additional peaks at 108 and $153 \mathrm{~cm}^{-1}$ for iodine-filled SWCNTs and a $192 \mathrm{~cm}^{-1}$ enhanced resonance, denoted $(12,5)^{\mathrm{E}}$, observed exclusively for CsI-filled SWCNTs. The 108 and $153 \mathrm{~cm}^{-1}$ resonances correspond to iodine trimers, $\mathrm{I}_{5}{ }^{-}$fragments, or $\mathrm{I}_{x}$ polymeric chains ${ }^{25}$ and are also observed at $660 \mathrm{~nm}$ (Figure S14a). Similar weak resonances are also seen in SWCNTs filled with CsI, indicating that a small amount of elemental iodine may have separated to create iodine filled SWCNTs, also observed in $\mathrm{NiI}_{2}$ filled SWCNTs. ${ }^{16}$ The $192 \mathrm{~cm}^{-1}$ resonance is exclusive to the CsI filled SWCNTs and conforms to an outlier semiconducting $(12,5)$ nanotube $^{40}$ with a diameter of $\sim 1.20 \mathrm{~nm}$, which would accommodate $2 \times 2 \mathrm{CsI}^{11}$ (see also Figure S13b). This indicates "tuning into resonance" similar to reported behavior for $2 \times 2 \mathrm{KI}$ crystals in $\sim 1.14 \mathrm{~nm}$ diameter SWCNTs. ${ }^{41}$ That this occurs in larger SWCNT for CsI may be a consequence of the larger diameter $\mathrm{Cs}^{+}$ion (i.e., $181 \mathrm{pm}$ versus $152 \mathrm{pm}$ for $\left.\mathrm{K}^{+}\right)^{42}$ which would incur a $\sim 8 \%$ expansion in the $2 \times 2$ CsI cross section, commensurate with an increase in the observed diameter. The results in Figures $6 \mathrm{a}$ and $\mathrm{S} 14 \mathrm{a}-\mathrm{c}$ can be taken as andication that the CNTs are filled, but a more detailed study of the influence of the filling on the RBM modes requires either microRaman on individual filled CNTs or a multiwavelength study on an ensemble of filled CNTs.

In Figure $6 \mathrm{~b}$, the OAS of unfilled SWCNTs show strong bands at 590-860 and 860-1300 nm corresponding to $\mathrm{E}_{22}^{\mathrm{S}}$ and $\mathrm{E}_{11}^{\mathrm{S}}$ excitonic transitions from numerous overlapping $(n, m)$ chiralities. $^{39,40}$ Following filling with CsI, most $\mathrm{E}_{11}^{\mathrm{S}}$ and $\mathrm{E}_{22}^{\mathrm{S}}$ absorbance features are unchanged apart from those for $(6,5)$ SWCNTs, which can only fill with linear -I-Cs-I-Cs-Ichains which show enhanced absorbance at 660 and $976 \mathrm{~nm}$, respectively. In $(7,5)$ and $(7,6)$ nanotubes, which fill with $2 \times 1$ CsI, $E_{11}$ and $E_{22}{ }_{22}$ absorbances at 660 and $1147 \mathrm{~nm}$ are still present. In nanotubes with diameters $>1.12 \mathrm{~nm}$, filling with $2 \times 2$ CsI is expected which theory predicts will $n$-dope the nanotubes. ${ }^{43}$ Literature values of the work function of each constituent can also be examined to assess the potential for charge transfer, i.e., $\sim 5 \mathrm{eV}$ for SWCNTs, ${ }^{44} \sim 5.5 \mathrm{eV}$ for iodine (bulk), ${ }^{45}$ and $3.4 \mathrm{eV}$ for CsI (bulk) ${ }^{46}$ with the latter two reducing with lowered dimensionality. ${ }^{47,48}$ Iodine therefore acts as an electron acceptor, receiving electrons from the SWCNT and making the nanotube $p$-type, ${ }^{41}$ whereas CsI should act as an electron donor and create $n$-type nanotubes. The degree of charge transfer was assessed from the absorption strength in the $\mathrm{E}^{\mathrm{S}}{ }_{11}$ range ${ }^{49,50}$ while the strong reduction in absorbance for iodine filling (Figure $6 \mathrm{~b}$ ) indicates a large shift in chemical potential. CsI filling does not noticeably alter the absorption 

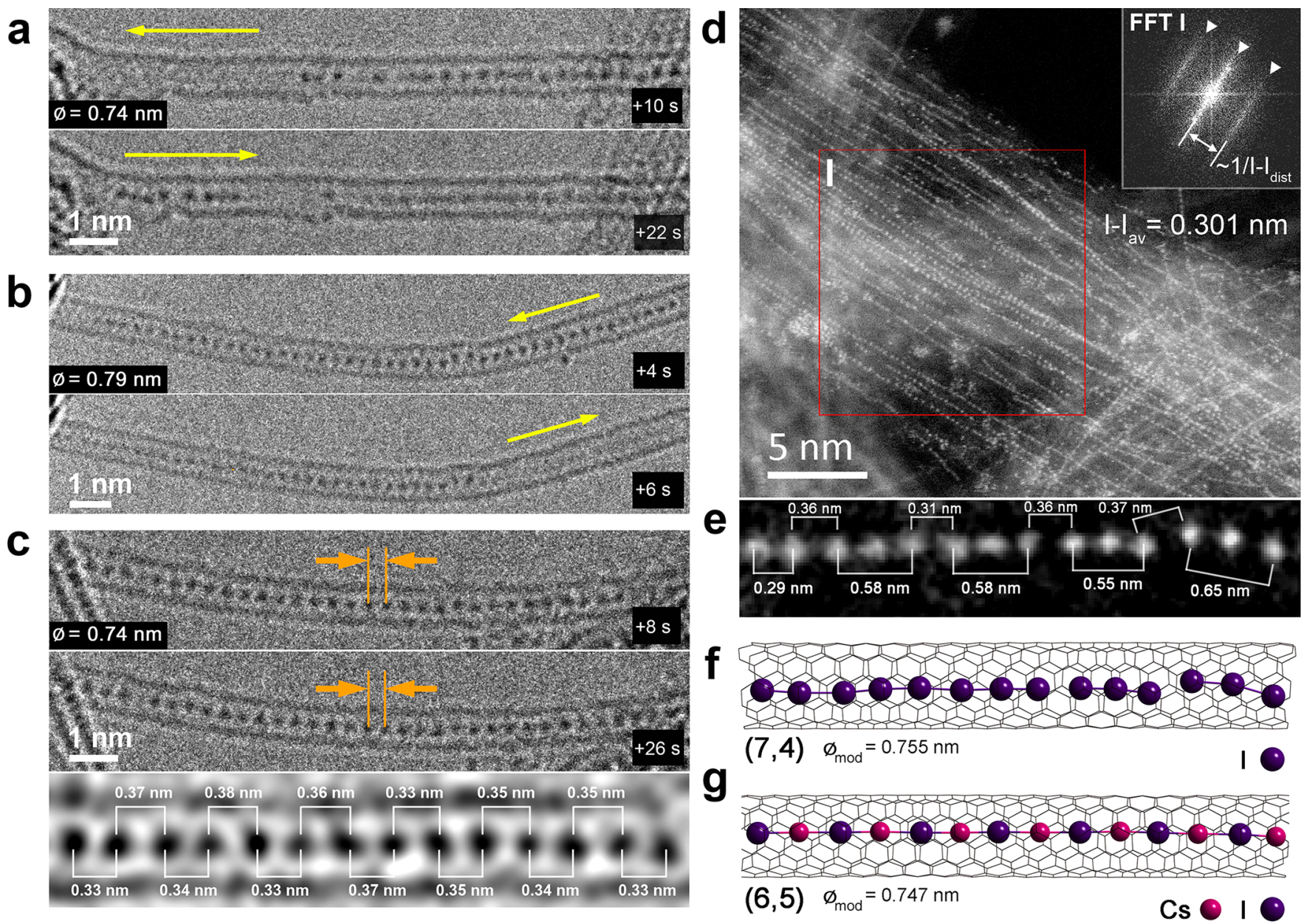

Figure 5. (a) Two images from an 18-image sequence CsI atoms in a partly filled straight SWCNT (0.74 diameter) showing disordered dynamical motion (yellow arrows, see also Movie S1). (b) Pair of images from a 20-image sequence in which canonical motion is observed in a partially filled curved $0.75 \mathrm{~nm}$ diameter SWCNT (yellow arrows, see also Movie S2) regulated by a defect near the center of the SWCNT. (c) Pair of images obtained 18s apart in 30-image sequence of continuously CsI-filled $0.74 \mathrm{~nm}$ diameter SWCNTs. In the animation, it is clear that the Cs and I atoms are rigidly fixed (orange arrows, see also Movie S3). A filtered, straightened region (bottom) shows a 14-atom CsI sequence with an average Cs-I separations of $0.35 \mathrm{~nm}$ but with variations from 0.33 to $0.38 \mathrm{~nm}$. (d) ADF-STEM image of an aligned bundle of SWCNTs containing exclusively 1D iodine chains. Inset FFT from boxed region I indicates an average I-I separation of $0.301 \mathrm{~nm} .{ }^{13}$ (e) ADF-STEM image of a discrete iodine chain forming trimers as reported in ref 15 with lengths indicated. The "stretched" trimer at right is an outlier. (f-g) Models of I and CsI chains embedded within appropriate diameter SWCNTs.

strength, suggesting small charge transfer, or a degree of charge compensation by a small parasitic I phase in the CsI samples (apparent in Figure 6a). A clearer view of the influence of CsI filling upon the optoelectronic properties of the SWCNTs was obtained from PL spectra (Figure 6c) of dispersed tubules in solution. Under optical excitation at $650 \mathrm{~nm}$, emission from $(8,3),(7,5)$, and $(7,6)$ chiralities was visible while $575 \mathrm{~nm}$ excitation probed $(6,5)$ and $(8,4)$ tubules. $^{39,40}$ Vertical solid lines indicate demarcation between "linear" $-\mathrm{I}-\mathrm{Cs}-\mathrm{I}-\mathrm{Cs}-\mathrm{I}-$ and $2 \times 1 \mathrm{CsI}$ filling and dashed lines indicate resonance wavelengths of unfilled SWCNTs. For $(6,5)$ SWCNTs filled with I-Cs-I-Cs-I-- chains the $\mathrm{E}^{\mathrm{S}}{ }_{11}$ emission was red-shifted with respect to the unfilled reference. The $(7,5),(7,6)$, and $(8,4)$ chiralities instead display blue-shifts when filled by $2 \times 1$ helical CsI. $\mathrm{E}^{\mathrm{S}}{ }_{11}$ emission was efficiently suppressed for all the iodinefilled $(n, m)$ tubules at both excitation wavelengths as a result of their $p$-type doping blocking radiative emission by phase space filling.

Figure 6d shows the full $2 \mathrm{D}$ photo-luminescence (PL) excitation for empty SWCNTs and CsI-filled SWCNTs, with the dashed lines indicating the profiles used in Figure 6c (these plots are reproduced in Figure S15 with the 2D plot for I@SWCNT added for comparison). Solid curves in the first two plots indicate onsets for linear $-\mathrm{I}-\mathrm{Cs}-\mathrm{I}-\mathrm{Cs}-, 2 \times 1$ and $2 \times 2 \mathrm{CsI}$ formation with widening SWCNT diameter. The PL plots for pristine SWCNTs indicate the predominating $(9,4)$ and $(7,6)$ semiconducting SWCNTs. After CsI filling, the distribution was dominated instead by $-\mathrm{I}-\mathrm{Cs}-\mathrm{I}-\mathrm{Cs}-$ filled $(6,5)$ SWCNTs, with relatively weaker emission from $(7,5),(9,4),(7,6)$, and $(8,4) 2 \times 1$-filled SWCNTs. Relatively weaker emission from the larger diameter $2 \times 1$-filled SWCNTs may result partially from small $n$-type doping. However, the oval distortion created by the helical $2 \times 1 \mathrm{CsI}$ filling could also disrupt the radiative emission from excitons, either by changing the intrinsic properties of the SWCNTs (e.g., electronic band structure, exciton binding energy) or by introducing disorder (e.g., fluctuations in tube diameters) on length scales shorter than the exciton diffusion length (typically $>100 \mathrm{~nm}$ ). 
a

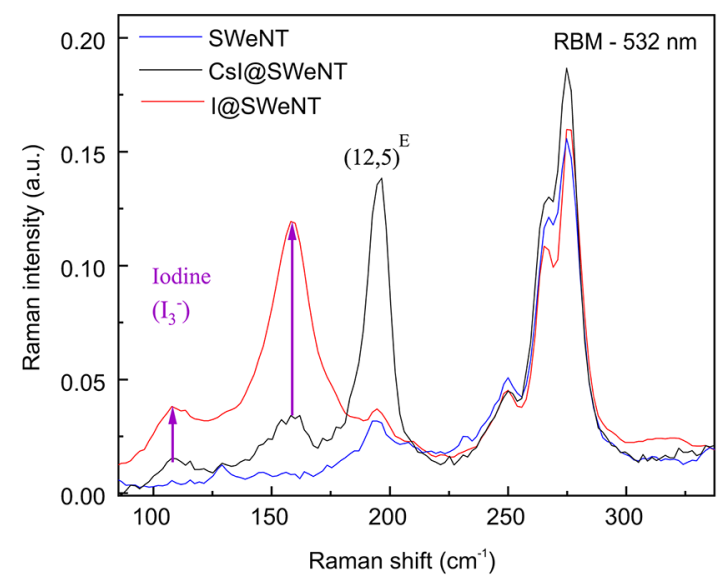

b

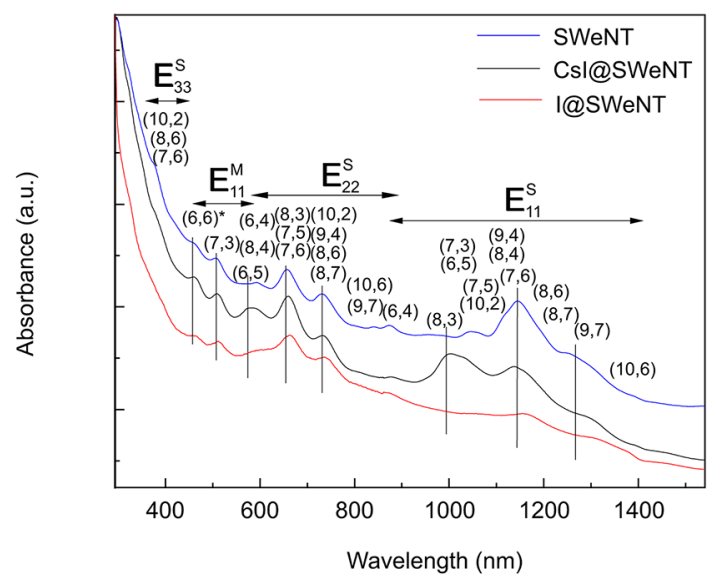

C
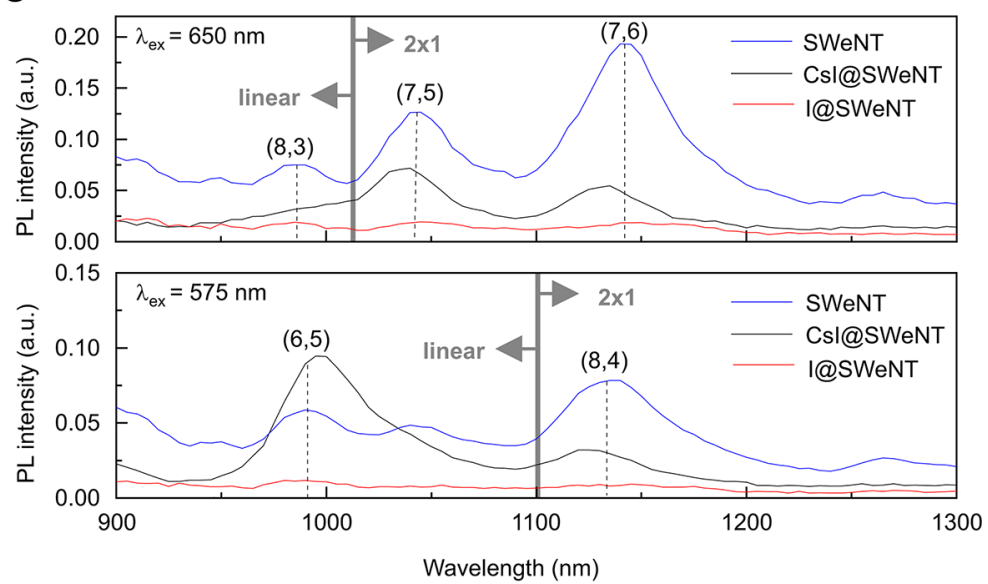

d

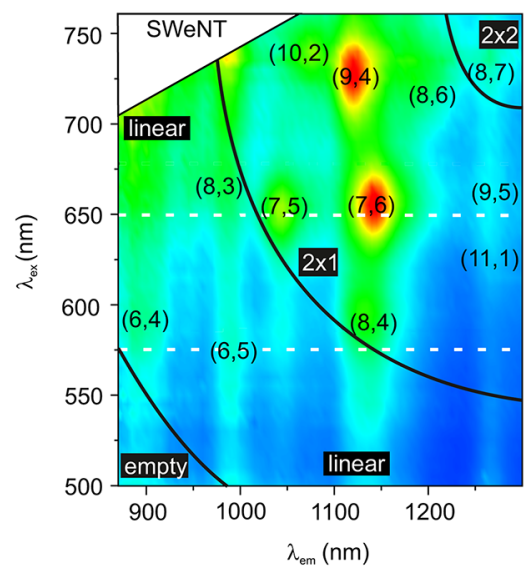

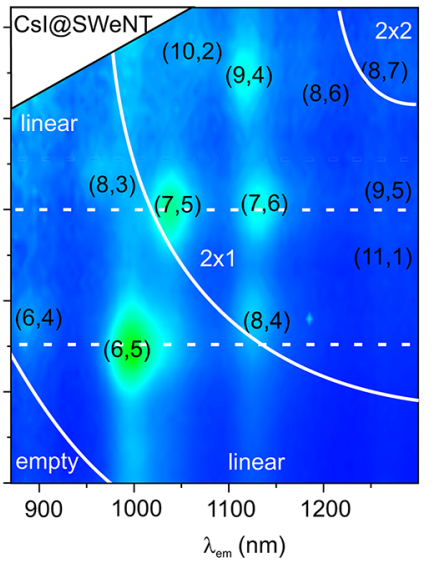

Figure 6. (a) Resonant RBMs of pristine SWCNTs, iodine, and CsI-filled SWCNTs at $532 \mathrm{~nm}$ laser excitation (660 nm excitation, Supporting Figure S14(a)). Raman shifts at 250, 266, and $275 \mathrm{~cm}^{-1}$ correspond to $(9,4),(7,6)$, and $(8,4)$ SWCNTs, respectively. Resonances due to polymeric iodine chains $\left(\mathrm{I}_{3}{ }^{-}, \mathrm{I}_{5}{ }^{-}\right.$, or continuous $\left.\mathrm{I}_{x}\right)$ in the iodine and CsI-filled SWCNTs and an enhanced SWCNT resonance (denoted $\left.(12,5)^{\mathrm{E}}\right)$ for the CsI-filled sample are visible between 100 and $200 \mathrm{~cm}^{-1}$. (b) Optical absorption spectra, normalized to the $\pi$-plasmon of empty SWCNTs, of empty SWCNTs, $I_{x}$ and CsI filled SWCNTS with $(n, m)$ peak assignments. ${ }^{40}$ Vertical lines indicate shifts in the peaks due to filling. (c), 900 to $1300 \mathrm{~nm}$ region from $(b)$ showing $\mathrm{E}_{11}^{\mathrm{s}}$ shifts with $(n, m)$ peaks indicated. Nearly all are suppressed for iodine-filled SWCNTs with only $(6,5)$ nanotubes having a significant absorbance and blue shift. CsI-filled $(7,5)$ and $(7,6)$ SWCNTs show prominent blue shifts, whereas CsI-filled (6,5) SWCNTs are unshifted. (d) 2D PL emission contour plots of empty, CsI and iodine-filled SWCNTs (left to right). The CsI-filled SWCNTs retain strong $(6,5)$ and blue-shifted $(7,5)$ and $(7,6)$ emissions, respectively, with nearly all $\mathrm{E}^{\mathrm{s}}{ }_{11}$ emissions for iodine- filled SWCNTs being quenched (see also Supporting Figure S15).

\section{CONCLUSIONS}

In this work, we reveal in detail the structural transformation of "True 1D" CsI atomic chains in narrow SWCNT with diameters from 0.73 to $0.81 \mathrm{~nm}$ to $2 \times 1 \mathrm{CsI}$ atomic chains in ovally distorting $\sim 0.84-0.95 \mathrm{~nm}$ diameter SWCNTs. For encapsulated $2 \times 1 \mathrm{CsI}$ helical chains, electron imaging reveals regulation of I-Cs-I angles by the SWCNT internal cross section and spectroscopic imaging confirms the chemical identify of linear $-\mathrm{I}-\mathrm{Cs}-\mathrm{I}-\mathrm{Cs}-$ chains versus $2 \times 1$ CsI helices, the latter forming in oval cross section SWCNTs. Raman, OAS, and PL optical measurements reveal the highly selective nature of doping that these ionic CsI chains produce in different narrow SWCNTs with $(6,5),(7,5)$, and $(7,6)$ all exhibit markedly different behavior. Our computational studies reveal the energetics of $2 \times 1 \mathrm{CsI}$ formation, and the resulting helical structure is found to be stable in the range $\sim 0.88$ to $1.08 \mathrm{~nm}$ but with $\mathrm{I}-\mathrm{Cs}-\mathrm{I}$ strain increasing as the SWCNT cross section reduces. These will be proportionally different for comparable alkali halide and other binary ionic systems but should demonstrate generality for linear or helical crystal growth inside smooth narrow tubules such as carbon nanotubes. Annealing helical structures with a specific $(n, m)$ conformation should also generate helices of constant pitch as their formation energy will reach a minimum for a specific diameter. These extended defects differ from the Schottky and Frenkel type normally found in alkali halides ${ }^{51}$ with longer range defects normally being stochastically induced by radiation or electron beam damage in thin halide films. ${ }^{52}$ By encapsulating, annealing and refining the diameter distribution of SWCNTs, we now have a genuine prospect of controlling the formation of helical chains in CsI and related materials with interesting consequences for encapsulate and composite physical properties as a result.

\section{METHODS}

Preparation of Csl- and $\mathrm{I}_{x}$-Filled SWCNTs for AC-TEM and ACADF-STEM. Bulk CsI (Sigma-Aldrich, 99.999\%) was vapor phase transported and deposited inside CoMoCAT SWeNT SWCNTs, originally supplied by SouthWest NanoTechnologies, with a specified diameter distribution of 0.7-1.1 nm (Product No. 404121). Filling of 
these nanotubes was effected via the sublimation method using similar conditions to those described elsewhere. ${ }^{13,18,21}$ Twenty milligrams of SWCNTs were first oxidized in open air at $750 \mathrm{~K}$ before being placed in one side of a $20 \mathrm{~cm}$ long two compartment silica quartz ampule with 50 mg CsI being placed at the end in the center of the hot zone of a Carbolite tube furnace. The hot end was heated to $825 \mathrm{~K}$ and the CsI was allowed to sublime along the natural temperature gradient created by the single hot zone of the furnace for 7 days into the preopened CoMoCAT SWCNTs maintained in the second compartment.

Electron Microscopy Imaging and Simulation. A doublecorrected JEM-ARM 200F microscope operating at $80 \mathrm{kV}$ equipped with CEOS imaging aberration and probe correction and a Gatan SC1000 ORIUS camera with a $4008 \times 2672$ pixel CCD was used for preliminary HRTEM and ADF STEM investigations. Electron beam densities were adjusted to between 0.8 to $1.88 \mathrm{pA} / \mathrm{cm}^{2}$. High-resolution high angle annular dark field (HAADF) imaging and electron energy loss spectroscopy (EELS) were carried out in a Nion UltraSTEM100 dedicated scanning transmission electron microscope (STEM), equipped with a Gatan Enfina spectrometer. The instrument was operated at $60 \mathrm{kV}$ acceleration voltage to minimize knock-on damage to the SWCNTs, and the STEM probe forming optics were adjusted to provide a $20 \mathrm{pA}$ beam with $33 \mathrm{mrad}$ convergence angle-resulting in an estimated probe size of approximately $1 \AA$. The EEL spectrometer entrance aperture half an gle acceptance was $36 \mathrm{mrad}$, while the HAADF detector inner and outer angles were 85 and $190 \mathrm{mrad}$, respectively. Chemical maps were produced from acquired EELS data by integrating the intensity of the relevant ionization edges (Cs and I $M_{4,5}$ ) over a $75 \mathrm{eV}$ window above their respective onsets, after subtraction of the decaying background using a standard power law model. A spectral dispersion of $0.5 \mathrm{eV} /$ channel was used to capture both edges simultaneously (resulting in a point-spread limited effective energy resolution of $\sim 1 \mathrm{eV}$ ); to further boost signal-to-noise the data was binned $(\times 2)$ spectrally prior to generating the EELS maps, but no statistical denoising was applied.

DFT and Bader Analysis Technical Details. All of the calculations are conducted with CASTEP-16.0 computational suite ${ }^{36}$ exploiting DFT with a plane-wave basis approach. Exchange and correlation was considered within a generalized gradient approximation in the form of the Perdew-Burke-Ernzerhof (PBE) functional. ${ }^{37}$ The core electrons were replaced by ultrasoft pseudopotentials while the electronic wave functions were expanded with a converged value for cutoff energy at $600 \mathrm{eV}$. All our models contain at least $12 \AA$ vacuum layers between periodic images that exclude spurious interactions. The Brillouin zone was integrated on a $1 \times 1 \times 11$ points of the Monkhorst and Pack grid. Geometry was optimized until forces on the atoms were smaller than $0.001 \mathrm{eV} / \AA$. The calculated charge was then processed with a grid-based Bader analysis. ${ }^{38}$ To account for possible effects of stress arising from incommensurate periodic models of CsI atomic chains and host CNTs, we include a stress compensation term in assessment of formation energy, which we find via calculations within "implicit nanotubes" where the repulsive electrostatic potential mimics steric confinement with CNTs. ${ }^{18}$

Raman, Optical Absorption, and Photoluminescence Spectroscopies. Optical absorbance spectroscopy was performed using a Lambda $1050 \mathrm{UV} /$ vis/IR spectrophotometer with a spectral range of 250-1500 nm. Raman spectra were obtained using confocal microspectrometers (Labram, Jobin-Yvon Horiba, and Renishaw inVia Reflex) with 660 and $532 \mathrm{~nm}$ excitation, a spectral resolution of 0.1 and $2 \mathrm{~cm}^{-1}$, and a spatial resolution of $1.5 \mu \mathrm{m}$. Photoluminescence spectra were measured by a Fluorolog UV/vis/IR fluorescence spectrophotometer (Horiba Jobin Yvon).

\section{ASSOCIATED CONTENT}

\section{(s) Supporting Information}

The Supporting Information is available free of charge at https://pubs.acs.org/doi/10.1021/acsnano.1c03705.

Information about image straightening procedures to used facilitate helical chain measurements; additional images and FFTs of CsI filling in SWCNT bundles; $a b$ initio modeling of CsI wires and SWCNT interactions including by DFT (CASTEP), force-field fitting by PotFit, PBE-DFT, SWCNT-ion interactions, LAMMPS simulations, Bader analysis; additional Raman measurements and additional PL data (PDF)

Movie of unsmooth motion of linear CsI chain in straight SWCNT (AVI)

Movie of unsmooth motion of linear CsI chain in curved SWCNT (AVI)

Movie of static linear CsI chain in continuosly filled SWCNT (AVI)

\section{AUTHOR INFORMATION}

\section{Corresponding Authors}

Reza J. Kashtiban - Department of Physics, University of Warwick, Coventry CV4 7AL, U.K.; ๑ orcid.org/00000002-3871-1647; Email: r.kashtiban@warwick.ac.uk

Andrij Vasylenko - Department of Physics, University of Warwick, Coventry CV4 7AL, U.K.; Department of Chemistry, University of Liverpool, Liverpool L69 7ZD, U.K.; ๑ orcid.org/0000-0002-6933-0628; Email: a.vasylenko@ liverpool.ac.uk

Jeremy Sloan - Department of Physics, University of Warwick, Coventry CV4 7AL, U.K.; ๑ orcid.org/0000-0001-86127456; Email: j.sloan@warwick.ac.uk

\section{Authors}

Maria G. Burdanova - Department of Physics, University of Warwick, Coventry CV4 7AL, U.K.

Jamie Wynn - Cavendish Laboratory, University of Cambridge, Cambridge CB3 OHE, U.K.

Paulo V. C. Medeiros - Cavendish Laboratory, University of Cambridge, Cambridge CB3 OHE, U.K.; ๑ orcid.org/00000002-7803-9058

Quentin Ramasse - SuperSTEM Laboratory, Daresbury WA44AD, U.K.; School of Chemical and Process Engineering, University of Leeds, Leeds LS2 9JT, U.K.; ๑ orcid.org/00000001-7466-2283

Andrew J. Morris - School of Metallurgy and Materials, University of Birmingham, Birmingham B15 2TT, U.K.; ๑ orcid.org/0000-0001-7453-5698

David Quigley - Department of Physics, University of Warwick, Coventry CV4 7AL, U.K.; ๑ orcid.org/0000-0003-47504372

James Lloyd-Hughes - Department of Physics, University of Warwick, Coventry CV4 7AL, U.K.; ๑ orcid.org/00000002-9680-0138

Complete contact information is available at: https://pubs.acs.org/10.1021/acsnano.1c03705

\section{Author Contributions}

J.S., R.J.K., M.B., A.V., P.V. C.M., A.J.M., D.Q., Q.R., and J.L.-H. conceived the project. R.J.K. performed the sample preparation and aberration corrected transmission electron microscopy characterization of filled nanotube samples with further imaging and analysis being carried out by J.S. and HAADF imaging and EELS being performed by Q.R. in Daresbury. Optical characterization of the samples was performed by M.B. and J.L.-H. Theoretical studies were performed by A.V., P.V.C.M., A.J.M., and D.Q. in Warwick and Birmingham. J.S., R.J.K., M.B., A.V., D.Q., and J.L.-H. wrote the manuscript and J.S. prepared the figures, including additional image processing, image simulation, 
and structure modeling with contributions from A.V., D.Q., R.J.K, Q.R., MB., and J.L-H. All authors discussed the results and commented on the manuscript.

\section{Notes}

The authors declare no competing financial interest.

Data used in this work are available via the Warwick data repository at wrap.warwick.ac.uk/156210.

\section{ACKNOWLEDGMENTS}

We are indebted to the EPSRC (UK) for support under Grant Nos. EP/N010825/1, EP/M011925/1, EP/M010643/1, and EP/R019428/1. M.G.B. thanks the Russian Government for financial support (Global Education program). We are also indebted to E. Hu for useful discussions regarding Raman and PL spectroscopy.

\section{REFERENCES}

(1) Eigler, D. M.; Schweizer, E. K. Positioning Single Atoms with a Scanning Tunneling Microscope. Nature 1990, 344, 524-526.

(2) Crommie, M. F.; Lutz, C. P.; Eigler, D. M. Confinement of Electrons to Quantum Corrals on a Metal Surface. Science 1993, 262, 218-220.

(3) Yeom, H. W.; Takeda, S.; Rotenberg, E.; Matsuda, I.; Horikoshi, K.; Schaefer, J.; Lee, C. M.; Kevan, S. D.; Ohta, T.; Nagao, T.; Hasegawa, S. Instability and Charge Density Wave of Metallic Quantum Chains on a Silicon Surface. Phys. Rev. Lett. 1999, 82, 4898-4901.

(4) Nadj-Perge, S.; Drozdov, I. K.; Li, J.; Chen, H.; Jeon, J.; MacDonald, A. H.; Bernevig, B. A. Observation of Majorana Fermions in Ferromagnetic Atomic Chains on a Superconductor. Science 2014, 346, 602-607.

(5) Lin, J.; Cretu, O.; Zhou, W.; Suenaga, K.; Prasai, D.; Bolotin, K. I.; Cuong, N. T.; Otani, Minoru; Okada, S.; Lupini, A. R.; Idrobo, J.-C.; Caudel, D.; Burger, A.; Ghimire, N. J.; Yan, J.; Mandrus, D. G.; Pennycook, S. J.; Pantelides, S. T. Metallic Nanowires with SelfAdaptive Contacts to Semiconducting Transition-Metal Dichalcogenide Monolayers. Nat. Nanotechnol. 2014, 9, 436-442.

(6) Lin, J.; Zhang, Y.; Zhou, W.; Pantelides, T. Structural Flexibility and Alloying in Ultrathin Transition-Metal Chalcogen Nanowires. ACS Nano 2016, 10, 2782-2790.

(7) Koh, A. L.; Wang, S.; Ataca, C.; Grossman, J. C.; Sinclair, R.; Warner, J. H. Torsional Deformations in Subnanometer MoS Interconnecting Wires. Nano Lett. 2016, 16, 1210-1217.

(8) Nagata, M.; Shukla, S.; Nakanishi, Y.; Liu, Z.; Lin, Y.-C.; Shiga, T.; Nakamura, Y.; Koyama, T.; Kishida, H.; Inoue, T.; Kanda, N.; Ohno, S.; Sakagawa, Y.; Suenaga, K.; Shinohara, H. Isolation of Single-Wired Transition-Metal Monochalcogenides by Carbon Nanotubes. Nano Lett. 2019, 19, 4845-4851.

(9) Sloan, J.; Kirkland, A. I.; Hutchison, J. L.; Green, M. L. H. Integral Atomic Architectures of 1D Crystals Inserted into Single Walled Carbon Nanotubes. Chem. Commun. 2002, 1319-1332.

(10) Fan, X.; Dickey, E. C.; Eklund, P. C.; Williams, K. A.; Grigorian, L.; Buczko, R.; Pantelides, S. T.; Pennycook, S. J. Atomic Arrangement of Iodine Atoms inside Single-Walled Carbon Nanotubes. Phys. Rev. Lett. 2000, 84, 4621-4624.

(11) Sloan, J.; Kirkland, A. I.; Hutchison, J. L.; Green, M. L. H. Aspects of Crystal Growth within Carbon Nanotubes. C. R. Phys. 2003, 4, 1063-1074.

(12) Fujimori, T.; Morelos-Gómez, A.; Zhu, Z.; Muramatsu, H.; Futamura, R.; Urita, K.; Terrones, M.; Hayashi, T.; Endo, M.; Hong, S. Y.; Choi, Y. C.; Tománek, D.; Kaneko, K. Conducting Linear Chains of Sulphur inside Carbon Nanotubes. Nat. Commun. 2013, 4, 2162/1-8.

(13) Senga, R.; Komsa, H.-P.; Liu, Z.; Hirose-Takai, K.; Krasheninnikov, A. V.; Suenaga, K. Atomic Structure and Dynamic Behaviour of Truly One-Dimensional Ionic Chains inside Carbon Nanotubes. Nat. Mater. 2014, 13, 1050-1054.
(14) Guan, L.; Suenaga, K.; Shi, Z.; Iijima, S. Polymorphic Structures of Iodine and Their Phase Transformation in Confined Nanospace. Nano Lett. 2007, 7, 1532-1535.

(15) Komsa, H.-P.; Senga, R.; Suenaga, K.; Krasheninnikov, A. V. Structural Distortions and Charge Density Waves in Iodine Chains Encapsulated inside Carbon Nanotubes. Nano Lett. 2017, 17, 36943700.

(16) Nie, C. Y.; Gabilert, A.-M.; Soula, B.; Datas, L.; Sloan, J.; Flahaut, E.; Monthioux, M. The Unexpected Complexity of Filling Double-Wall Carbon Nanotubes with Nickel (and Iodine) 1-D Nanocrystals. IEEE Trans. Nanotechnol. 2017, 16, 759-766.

(17) Shi, L.; Rohringer, P.; Suenaga, K.; Niimi, Y.; Kotakoski, J.; Meyer, J. C.; Peterlik, H.; Wanko, M.; Cahangirov, S.; Rubio, A.; Lapin, Z. J.; Novotny, L.; Ayala, P.; Pichler, T. Confined Linear Carbon Chains as a Route to Bulk Carbyne. Nat. Mater. 2016, 15, 634-639.

(18) Medeiros, P. V. C.; Marks, S.; Wynn, J. M.; Vasylenko, A.; Ramasse, Q. M.; Quigley, D.; Sloan, J.; Morris, A. J. Single-Atom Scale Structural Selectivity in Te Nanowires Encapsulated inside Ultranarrow Single-Walled Carbon Nanotubes. ACS Nano 2017, 11, 6178-6185.

(19) Hart, M.; White, E. R.; Chen, J.; McGilvery, C. M.; Pickard, C. J.; Michaelides, A.; Sella, A.; Shaffer, M. S. P.; Salzmann, C. G. Enapsulation and Polymerization of White Phosphorous inside Single-Walled Carbon Nanotubes. Angew. Chem., Int. Ed. 2017, 56, 8144-8148.

(20) Hart, M.; Chen, J.; Michaelides, A.; Sella, A.; Shaffer, M. S. P.; Salzmann, C. G. One-Dimensional Arsenic Allotropes: Polymerization of Yellow Arsenic inside Single-Wall Carbon Nanotubes. Angew. Chem., Int. Ed. 2018, 57, 11649-11653.

(21) Vasylenko, A.; Marks, S.; Wynn, J. M.; Medeiros, P. V. C.; Ramasse, Q. M.; Morris, A. J.; Sloan, J.; Quigley, D. Electronic Structure Design of Sub-Nanometre 1D SnTe via Nanostructuring within SingleWalled Carbon Nanotubes. ACS Nano 2018, 12, 6023-6031.

(22) Slade, C. A.; Sanchez, A. M.; Sloan, J. Unprecedented New Crystalline Forms of SnSe in Narrow to Medium Diameter Carbon Nanotubes. Nano Lett. 2019, 19, 2979-2984.

(23) Senga, R.; Suenaga, K. Single-Atom Detection of Light Elements: Imaging or Spectroscopy ? Ultramicroscopy 2017, 180, 150-155.

(24) Grigorian, L.; Williams, K. A.; Fang, S.; Sumanasekera, G. U.; Loper, A. L.; Dickey, E. C.; Pennycook, S. J.; Eklund, P. C. Reversible Intercalation of Charged Iodine Chains into Carbon Nanotube Ropes. Phys. Rev. Lett. 1998, 80, 5560-5563.

(25) Chorro, M.; Kané, G.; Alvarez, L.; Cambedouzou, J.; Paineaud, E.; Rossberge, A.; Kociak, M.; Aznar, R.; Pascarellia, S.; Launois, P.; Bantignies, J. L. 1D Confinements of Polyiodides inside Single-Walled Carbon Nanotubes. Carbon 2013, 52, 100-108.

(26) Tonkikh, A. A.; Obraztsova, E. D.; Obraztsova, E. A.; Belkin, A. V.; Pozharov, A. S. Optical Spectroscopy of Iodine-Doped Single-Wall Carbon Nanotubes of Different Diameter. Phys. Status Solidi B 2012, B249, 2454-2459.

(27) Poborchii, V. V. Polarized Raman and Optical Absorption Spectra of the Mordenite Single Crystals Containing Sulfur, Selenium or Tellurium in the One-Dimensional Nanochannels. Chem. Phys. Lett. 1996, 251, 230-234.

(28) Poborchii, V. V.; Kolobov, A. V.; Caro, J.; Zhuravlev, V. V.; Tanaka, K. Dynamics of Single Selenium Chains Confined in OneDimensional Nanochannels of $\mathrm{AlPO}_{4}-5$ : Temperature Dependencies of the First- and Second-Order Raman Spectra. Phys. Rev. Lett. 1999, 82, 1955-1958.

(29) Ivanov, V. G.; Kalashnyk, N.; Sloan, J.; Faulques, E. Vibrational Dynamics of Extreme $2 \times 2$ and $3 \times 3$ Potassium Iodide Nanowires Encapsulated in Single-Walled Carbon Nanotubes. Phys. Rev. B: Condens. Matter Mater. Phys. 2018, 98, 125429.

(30) Spencer, J. H.; Nesbitt, J. M.; Trewhitt, H.; Kashtiban, R. J.; Bell, G.; Ivanov, V. G.; Faulques, E.; Sloan, J.; Smith, D. C. Raman Spectroscopy of Optical Transitions and Vibrational Energies of $\sim 1$ $\mathrm{nm}$ HgTe Extreme Nanowires within Single Walled Carbon Nanotubes. ACS Nano 2014, 8, 9044-9052.

(31) Ilie, A.; Bendall, J. S.; Roy, D.; Philp, E.; Green, M. L. H. Effects of KI Encapsulation in Single-Walled Carbon Nanotubes by Raman and 
Optical Absorption Spectroscopy. J. Phys. Chem. B 2006, 110, 1384813857.

(32) Vasylenko, A.; Wynn, J.; Medeiros, P. V. C.; Morris, A. J.; Sloan, J.; Quigley, D. Encapsulated Nanowires: Boosting Electron Transport in Carbon Nanotubes. Phys. Rev. B: Condens. Matter Mater. Phys. 2017, 95, 121408 .

(33) Kharlamova, M. V.; Kramberger, C.; Rudatis, P.; Pichler, T.; Eder, D. Revealing the Doping Effect of Encapsulated Lead Halogenides on Single-Walled Carbon Nanotubes. Appl. Phys. A: Mater. Sci. Process. 2019, 125, 320.

(34) Philp, E.; Sloan, J.; Kirkland, A. I.; Meyer, R. R.; Friedrichs, S.; Hutchison, J. L.; Green, M. L. H. An Encapsulated Helical 1D Cobalt Iodide Crystal. Nat. Mater. 2003, 2, 788-791.

(35) Hirahara, K.; Suenaga, K.; Bandow, S.; Kato, H.; Okazaki, T.; Shinohara, H.; Iijima, S. One-Dimensional Metallofullerene Crystal Generated inside Single-Walled Carbon Nanotubes. Phys. Rev. Lett. 2000, 85, 5384-5387.

(36) Clark, S. J.; Segall, M. D.; Pickard, C. J.; Hasnip, P. J.; Probert, M. J.; Refson, K.; Payne, M. C. First Principles Methods Using CASTEP. Z. Kristallogr. - Cryst. Mater. 2005, 220, 567-570.

(37) Perdew, J. P.; Burke, K.; Ernzerhof, M. Generalized Gradient Approximation Made Simple. Phys. Rev. Lett. 1996, 77, 3865-3868.

(38) Tang, W.; Sanville, W. E.; Henkelman, G. A Grid-Based Bader Analysis Algorithm without Lattice Bias. J. Phys.: Condens. Matter 2009, 21,084204 .

(39) Christian, T.; Reich, S. In Raman Scattering in Carbon Nanotubes" in Light Scattering in Solids IX; Cardona, M., Merlin, R., Eds.; Springer: Berlin, Heidelberg, 2007; pp115-234.

(40) Weisman, R. B.; Bachilo, S. M. Dependence of Optical Transition Energies on Structure for Single-Walled Carbon Nanotubes in Aqueous Suspension: An Empirical Kataura Plot. Nano Lett. 2003, 3, 12351238 .

(41) Ilie, A.; Bendall, J. S.; Roy, D.; Philp, E.; Green, M. L. H. Effects of KI Encapsulation in Single-Walled Carbon Nanotubes by Raman and Optical Absorption Spectroscopy. J. Phys. Chem. B 2006, 110, 1384813857.

(42) Mann, J. B. Atomic Structure Calculations II. Hartree-Fock Wave Functions and Radial Expectation Values: Hydrogen to Lawrencium, LA3691; Los Alamos Scientific Laboratory, USA, 1968.

(43) Bichoutskaia, E.; Pyper, C. Theoretical Study of the Structures and Electronic Properties of All-Surface KI and CsI Nanocrystals Encapsulated in Single Walled Carbon Nanotubes. J. Chem. Phys. 2008, $129,154701$.

(44) Shiraishi, M.; Ata, M. Work Function of Carbon Nanotubes. Carbon 2001, 39, 1913-1917.

(45) Yamamoto, H. Ultraviolet Photoelectron Spectroscopy of Solid Iodine. J. Chem. Phys. 1987, 86, 1775-1779.

(46) Verma, R. L. Potential and Limitations of Cesium Iodide as a Dynode Material for Use in Electron Multipliers. J. Phys. D: Appl. Phys.

1978, 11, 63-71.

(47) Avery, N. R. LEED, Auger and Work Function Study of Iodine Adsorbed on W (100). Surf. Sci. 1974, 43, 101-122.

(48) Fairchild, S. B.; Back, T. C.; Murray, P. T.; Cahay, M. M.; Shiffler, D. A. Low Work Function CsI Coatings for Enhanced Field Emission Properties. J. Vac. Sci. Technol., A 2011, 29, 031402.

(49) Kazaoui, S.; Minami, N.; Jacquemin, R.; Kataura, H.; Achiba, Y. Amphoteric Doping of Single-Wall Carbon-Nanotube Thin Films as Probed by Optical Absorption Spectroscopy. Phys. Rev. B: Condens. Matter Mater. Phys. 1999, 60, 13339-13342.

(50) Burdanova, M. G.; Tsapenko, A. P.; Satco, D. A.; Kashtiban, R.; Mosley, C. R.; Monti, M.; Staniforth, M.; Sloan, J.; Gladush, Y. G.; Nasibulin, A. G.; Lloyd-Hughes, J. Giant Negative Terahertz Photoconductivity in Controllably Doped Carbon Nanotube Networks. ACS Photonics 2019, 6, 1058-1066.

(51) Royce, B. S. H. The Creation of Point Defects in Alkali Halides. Prog. Solid State Chem. 1967, 4, 213-243.

(52) Catlow, C. R. A.; Diller, K. M.; Hobbs, L. W. Irradiation-Induced Defects in Alkali Halide Crystals. Philos. Mag. A 1980, 42, 123-150. 